\title{
Risk of Systemic Infections Associated with Umbilical Vessel Catheterization
}

\author{
Takuji Kumagai and Akira Watanabe \\ Department of Pediatrics, Sapporo Medical College, Sapporo \\ 060, and Department of Pediatrics, Aomori Prefectural Central \\ Hospital, Aomori 030
}

\begin{abstract}
Kumagai, T. and Watanabe, A. Risk of Systemic Infections Associated with Umbilical Vessel Catheterization. Tohoku J. exp. Med., 1978, 125 (2), 155-162 - The purpose of the present study is to determine the risk of infections associated with umbilical vessel catheterization. During a period of recent four years, 97 catheters were inserted into the umbilical vessels of 94 newborn infants in a newborn nursery. Thirty percent of the catheter tips were colonized upon removal with pathogens $(11 \%)$ and contaminants. The rate of catheter colonization was not dependent on sex, gestational age, birth weight and duration of catheterization. Bacteria were isolated from blood specimen drawn via the catheter upon removal in one patient, but blood sample from peripheral artery and catheter tip yielded no organisms in this patient. One patient $(1 \%)$ was found to be septicemic while the catheter was in place. Culture of the catheter tip and blood drawn via the catheter proved unreliable in the diagnosis of subsequent systemic infection. Judicious surveillance for systemic infection by peripheral blood culture is necessary. - umbilical vessel catheterization; neonatal systemic infection
\end{abstract}

In recent years, the usefulness of indwelling umbilical vessel catheter has been established and it has become a common procedure in the management of the acutely ill and high-rsik newborn infants. The convenience and advantages of umbilical vessel catheter as stable and durable intravascular route for infusion, monitoring acid-base status and exchange transfusion are obvious as compared with other methods such as scalp vein needle, venous cut down and frequent puncture of peripheral artery. However, there are many reports of complications such as thrombosis, embolization, hemorrhage and infection (Kitterman et al. 1970). We have paid attention to the risk of systemic infections associated with umbilical vessel catheterization, because the umbilicus is almost heavily contaminated at the time of catheter insertion (Fairchild et al. 1958) and newborn infants are particularly prone to infection. This report describes the results of prospective study in newborn patients to define the risk of systemic infection associated with umbilical vessel catheterization. There have been few studies on this problem in

Received for publication, October 22, 1977.

Presented before the 80th Annual Session of the Japanese Society of Pediatrics, Sapporo, June 21-23, 1977. 
our country, and so far as we know, this paper is the first report from Japan in this area.

\section{Materials and Methods}

The subjects of the present study were 94 infants who had umbilical catheterization in the newborn nursery of the Aomori Prefectural Central Hospital from April 1, 1973 to March 31, 1977. All the infants studied were born elsewhere and referred to us because of severe neonatal jaundice, respiratory distress and others. Thirty-eight infants were full term (gestational age; over 37 weeks) and 56 were preterm infants. The apparent discrepancy in total numbers of catheters and patients is due to the fact that more than one catheter were inserted into some infants. The birth weight was over $2500 \mathrm{~g}$ in 30 infants, 1500 to $2500 \mathrm{~g}$ in 37 , and below $1500 \mathrm{~g}$ in the remaining 27 . All catheterizations were performed by members of house staff. The umbilicus and surrounding skin of the infant were washed first with tincture of iodine followed by $70 \%$ alcohol. The umbilical area was kept sterile by a sterile circumcision sheet exposing only the umbilical stump. The operator always used a sterile gown and gloves. The cord was cut $0.5-1.5 \mathrm{~cm}$ from the skin-umbilical cord junction, and the radiopaque polyvinyl chloride catheter (end -and-sidehole type, No. 5 or 3.5 French) was inserted into the umblical vessel. The catheter was then fixed in place with silk thread. A purse-string suture was also placed in Wharton's jelly around the vessel and catheter. The area was covered with a $5 \times 5 \mathrm{~cm}$ sterile dry gauze dressing, over which one or two loops of catheter were secured with paper tape. Topical antibiotics was not used. The dressing was not changed until removal of the catheter. Catheter distance were predetermined from graphs prepared from Dunn's postmortem measurements (Dunn 1966). Final positions were confirmed by anteroposterior and lateral roentgenograms of the chest and abdomen. Infusion was maintained with a constant infusion pump. The indications for placement of catheters in this series were respiratory distress $(48 \%)$, severe neonatal jaundice $(28 \%)$, congenital heart disease $(6 \%)$ and others $(18 \%)$. Systemic antibotics were not routinely administered to all patients with umbilical vessel catheterization, and the patients who were administered systemic antibiotics were excluded from this study because the duration and route of administration, and doses and sort of antibiotics were not controlled. When conditions allowed of removal of the eatheter, the following procedures were performed according to the study protocol. First, a peripheral blood culture was obtained from the radial artery after sterilizing the skin with alcohol. A blood sample was then obtained via the umbilical catheter (cord blood) for culture. Finally, the catheter was then carefully withdrawn to avoid contamination and the catheter tip was smeared on the McConkey's and blood agar plates. The catheter tip cultures were read at 24 and $48 \mathrm{hr}$ and blood cultures were examined daily for at least 10 days. All cultures were processed by the Diagnostic Bacteriology Laboratory and the standard method for indentification of aerobic and anaerobic organisms were employed. If an infant died, the catheter was always left in situ so that changes related to the catheter could be seen at postmortem examination. Catheter tip cultures were done in the autopsy room by the same manner as mentioned above. Blood cultures were obtained by cardiac puncture. Pertinent clinical data were recorded on each patient. These included birth weight, age of gestation, sex differentiation, and duration of catheterization. A comparison was made between these parameters and the results of cultures to determine some consistent relationship.

\section{RESUlts}

Some of the 94 patients had both vein- and artery-catheterization concurrently or serially, and a total of 97 catheters, 33 arterial and 64 venous, were placed in 94 patients. Twenty-three infants died, and autopsies were performed in $78 \%$ of them. Complications probably related to the umbilical vessel catheteriza- 
TABLE 1. Organisms recovered from culture of catheter tip

\begin{tabular}{lll}
\hline \multirow{2}{*}{ Organism } & \multicolumn{2}{c}{ No. of isolates } \\
\cline { 2 - 3 } & Artery & Vein \\
\cline { 2 - 3 } Pathogen; & & \\
S. aureus & 1 & 4 \\
E. coli & 0 & 1 \\
Pseudomonas & 0 & 1 \\
Proteus & 1 & 1 \\
Enterobacter & 0 & 1 \\
Enterococeus & 0 & 1 \\
Contaminant; & & \\
S. epidermidis & 1 & 2 \\
Mima polymorpha & 0 & 3 \\
Others & 4 & 8 \\
\hline
\end{tabular}

tion were found in 2 of the 18 autopsy cases. In the remaining 16 cases, no complication was found.

A total of 97 catheter tip cultures were obtained from the babies studied. Seven of 33 arterial catheter tip cultures $(21 \%)$ and 22 of 64 venous catheter tip cultures $(34 \%)$ demonstrated bacterial growth. The other 70 catheters were negative.

Table 1 shows the type of bacteria isolated from catheter tips in both umbilical vessels. The organisms isolated were divided into the so-called pathogens (coagulase-positive staphylococcus, Escherichia coli, pseudomonas, proteus, enterobacter, and enterococcus) and the contaminants (coagulase-negative staphylococcus, Mima polymorpha, and others) based on the studies by Zinner et al. (1969), Nordin (1969), and Balagtas et al. (1971). Eleven percent of the catheter tips were colonized with pathogens and $19 \%$ with contaminants by this classification. The most frequently isolated organisms were coagulase-negative and coagulasepositive staphylococci, which accounted for $28 \%$ of all the positive cultures. In all of the instances the positive catheter tip cultures yielded a single bacterial genus. There was no statistical difference either in colonization rate or in the incidence of pathogens and contaminants between arterial and venous routes.

The sex distribution in relation to the results of umbilical tip cultures in artery and vein are shown in Table 2 . The sex of the newborn infants did not affect the colonization rate in both vessels ( $\chi^{2}$ with Yates' correction; 0.86 in artery, 0.11 in vein, $p>0.25$ ). The colonization rate of arterial catheter tip in females appeared to be low as compared with that in males; however, this difference was not

TABLE 2. Results of Catheter tip clutures and sex distribution

\begin{tabular}{ccccc}
\hline Umbilical vessel & Result of culture & No. of cases & Male & Female \\
\hline \multirow{2}{*}{ Artery } & + & 7 & 6 & 1 \\
\multirow{2}{*}{ Vein } & - & 26 & 15 & 11 \\
& + & 22 & 13 & 9 \\
& - & 42 & 23 & 19 \\
\hline
\end{tabular}


TABLE 3. Results of catheter tip culture and birth weight of the patients

\begin{tabular}{crrrrrr}
\hline \multirow{2}{*}{ Birth weight $(\mathrm{g})$} & \multicolumn{3}{c}{ Artery } & \multicolumn{2}{c}{ Vein } & Total \\
\cline { 2 - 5 } & + & - & + & - & \\
\hline$<1500$ & 4 & 12 & 4 & 9 & 29 \\
$1500-2500$ & 3 & 10 & 5 & 20 & 38 \\
$\geq 2500$ & 0 & 4 & 13 & 13 & 30 \\
\hline
\end{tabular}

TABLE 4. Results of catheter tip cultures and gestational age of the patients

\begin{tabular}{crrrrrr}
\hline $\begin{array}{c}\text { Gestational } \\
\text { age (week) }\end{array}$ & \multicolumn{2}{c}{ Artery } & \multicolumn{2}{c}{ Vein } & Total \\
\cline { 2 - 5 } & + & - & + & - & \\
\hline 30 & 3 & 11 & 4 & 11 & 29 \\
$31-36$ & 3 & 10 & 6 & 11 & 30 \\
$\geqq 37$ & 0 & 6 & 13 & 19 & 38 \\
\hline
\end{tabular}

TABLE 5. The yearly change of average indwelling time of umbilical catheter

\begin{tabular}{ccc}
\hline Year & Artery & Vein \\
\hline & days & days \\
1973 & - & 4.9 \\
1974 & 5.2 & 5.4 \\
1975 & 3.7 & 3.4 \\
1976 & 5.0 & 1.7 \\
1977 & 4.1 & 0.75 \\
\hline
\end{tabular}

statistically significant.

Table 3 shows the birth weight in relation to the results of umbilical tip cultures in artery and vein. No correlation was found between birth weight and the incidence of positive tip cultures in both vessels $\left(\chi^{2}=1.24\right.$ in artery, $p>0.25$; 5.18 in vein, $0.02<p<0.025$ )

The gestational age in relation to the results of umbilical tip cultures in artery and vein are shown in Table 4. There was no significant difference in the frequency of positive cultures among the three groups of different gestational age in both vessels $\left(\chi^{2}=1.64\right.$ in artery, 0.87 in vein, $\left.p>0.20\right)$.

Age at the time of catheterization ranged from $30 \mathrm{~min}$ to 7 days. The duration of catheterization was 2 to 4 days in $32 \%$ of the cases, more than 4 days in $40 \%$, and less than $48 \mathrm{hr}$ in $28 \%$. The longest was 12 days of serial 2 catheters in one patient. The average indwelling time was $104 \mathrm{hr}$ in artery and $92 \mathrm{hr}$ in vein, and there was definite trend to diminish the duration of catheterization year by year (Table 5).

Table 6 shows the duration of catheterization in relation to the results of umbilical tip cultures in artery and vein. The rate of catheter colonization was 
TABLE 6. Results of catheter tip cultures and duration of catheterization

\begin{tabular}{|c|c|c|c|c|c|}
\hline \multirow{2}{*}{ Duration (hr) } & \multicolumn{2}{|c|}{ Artery } & \multicolumn{2}{|c|}{ Vein } & \multirow{2}{*}{ Total } \\
\hline & + & - & + & - & \\
\hline$\leqq 48$ & 1 & 6 & 7 & 13 & 27 \\
\hline $49-72$ & 2 & 5 & 3 & 10 & 20 \\
\hline $73-96$ & 1 & 4 & 2 & 4 & 11 \\
\hline $97-120$ & 0 & 4 & 4 & 5 & 13 \\
\hline$\geqq 121$ & 3 & 7 & 6 & 10 & 26 \\
\hline
\end{tabular}

TABLE 7. The incidence of pathogen and contaminant recovered from the catheter tip

\begin{tabular}{lrrrrrrl}
\hline & \multicolumn{6}{c}{ Days of catheterization } & \multirow{2}{*}{ Total } \\
\cline { 2 - 6 } Result of culture & $\leqq 2$ & $2-3$ & $3-4$ & $4-5$ & $5<$ & \\
\hline No growth & 19 & 15 & 8 & 9 & 17 & $68(70.1 \%)$ \\
"Contaminant" & 6 & 1 & 1 & 3 & 8 & $19(19.69)$ \\
"Pathogen" & 2 & 4 & 2 & 1 & 1 & $10(10.3 \%)$ \\
$\quad$ Total & 27 & 20 & 11 & 13 & 26 & 97
\end{tabular}

not dependent on duration of catheterization in both vessels $\left(\chi^{2}=1.97\right.$ in artery, 1.22 in vein, $p>0.10$ ).

The incidence of pathogens and contaminants recovered from the catheter tips in relation to the duration of catheterization are shown in Table 7. The duration of catheterization did not influence the incidence of pathogens and contaminants cultured $\left(x^{2}=8.61\right.$, D.F. $\left.=8, p>0.20\right)$.

A total of 19 pairs of cultures of the cord blood and peripheral blood obtained from the radial artery in 18 patients were processed. Nine patients had arterial catheterization and 9 had venous catheterization. Staphylococcus edpidermidis was recovered from the cord blood of one infants $(5 \%)$, but the blood from the radial artery and the catheter tip were negative in this patient. This infant was full-term and had venous catheterization for $40 \mathrm{hr}$ because of severe neonatal jaundice. The patient was clinically well and did not develop septicemia. Eighteen cord blood cultures from 17 other infants remained sterile. No blood sample taken from the radial artery yielded any organisms in these cases.

A serious complication, causing or contributing to death, was found at autopsy in two patients as mentioned before. A septic complication occurred in only one infant (1\%), a preterm baby who had umbilical artery catheterization for respiratory distress syndrome. Staphylococcus aureus was recovered from the catheter tip and blood obtained from the radial artery. Cord blood culture was not done in this case. The autopsy showed abscesses in the lungs and liver. Another patient in whom the catheter was the likely cause of death was a full-term baby with Down's syndrome and had umbilical venous catheterization for severe neonatal jaundice. Periumbilical abscess occurred in this patient 3 days after withdrawal 
of the catheter, and symptoms of peritonitis developed. The patient recovered once; however, death occurred at age of 4 months due to ileus. The autopsy revealed strangulation ileus of the jejunum. Catheter tip culture was negative in this patient.

\section{Discussion}

It is well known that the umbilical catheter provides a frequent portal of entry for bateria into the blood of the newborn. During the past several years, numerous reports have attested to the hazards of prolonged umbilical vessel catheterization. Many authors investigated the risk of systemic infection associated with umbilical vessel catheterization by using umbilical catheter tip cultures. Table 8 shows the reported experiences of tip cultures by other authors. The colonization rate reported by Krauss et al. (1970) and Symansky and Fox (1972) represents two ends of spectrum in non-antibiotics group of infants. The reported incidence varied widely. In our study the incidence of positive catheter tip lies between the two ends of spectrum. As mentioned in other studies, no relation was found between the the incidence of bacterial contamination and several parameters such as duration of catheterization, gestational age and birth weight of infants. The influence of systemic antibiotics on catheter colonization rate was not definite and stable in other studies (Krauss et al. 1970; Balagtas et al. 1971; Powers and Tooley 1972; Bard et al. 1973; Anagnostakis et al. 1975). Powers and Tooley (1972) stated that the incidence of contamination of umbilical vessel catheter varies in accordance with the technique used as well as the care and experience of the personnel. Balagtas et al. (1971) also stated that contamination of catheter occurs regularly during catheter insertion with organism from the cord stump which is colonized with

TABLE 8. Reported experiences of catheter tip culture

\begin{tabular}{|c|c|c|c|c|}
\hline \multirow[t]{2}{*}{ Authors } & \multirow{2}{*}{$\begin{array}{l}\text { Antibiotics } \\
\text { employed }\end{array}$} & \multirow{2}{*}{$\begin{array}{l}\text { Artery or } \\
\text { vein }\end{array}$} & \multicolumn{2}{|c|}{$\begin{array}{c}\text { Percentage of positive catheter tip } \\
\text { culture }\end{array}$} \\
\hline & & & A.B $(+)$ & A.B (一) \\
\hline Krauss 1970 & $\mathrm{ABPC}+\mathrm{KM}$ & $A \& V$ & $70.0 \%$ & $75.0 \%$ \\
\hline Casalino 1971 & $\mathrm{ABPC}+\mathrm{KM}$ & $A \& V$ & 5.0 & \\
\hline Balagtas 1791 & $\mathrm{ABPC}+\mathrm{KM}$ & V & 33.3 & 70.5 \\
\hline Johns 1972 & & $A \& V$ & & 10.9 \\
\hline Symansky 1972 & & A & & 6.3 \\
\hline & $\triangle \mathrm{RPC} \perp \mathrm{KM}$ & $\begin{array}{l}V \\
A\end{array}$ & & 2.3 \\
\hline $\begin{array}{l}\text { Powers } 1972 \\
\text { Bard } 1973\end{array}$ & $\begin{array}{l}\mathrm{ABPC}+\mathrm{KM} \\
\mathrm{ABPC}+\mathrm{KM}\end{array}$ & $\begin{array}{l}A \\
\mathrm{~A}\end{array}$ & 9.6 & 7.6 \\
\hline Daschner 1974 & & $\begin{array}{l}\mathrm{A} \\
\mathrm{A}\end{array}$ & 24.2 & $\begin{array}{r}54.3 \\
3.1\end{array}$ \\
\hline & & V & & 23.4 \\
\hline Franke 1974 & $\mathrm{ABPC}, \mathrm{CET}+\mathrm{CL}$ & $\mathbf{A}$ & 5.5 & \\
\hline Anagnostakis 1975 & $\mathrm{PC}+\mathrm{KM}$ & $\mathbf{V}$ & 60.0 & 63.0 \\
\hline Present study 1977 & & $A$ & & 21.2 \\
\hline & & V & & 34.3 \\
\hline
\end{tabular}

A.B $(+)$; Antibiotics treated group

A.B (-); Antibiotics non-treated group 
bacteria soon after birth. In this regard, it is now suggested that catheter tip colonization is worthless by itself in evaluation of bacterial infection of infants. The diagnosis of neonatal septicemia should be ultimately based on a positive blood culture.

Cowett et al. (1976) compared the colonization rate of peripheral blood with those obtained from the umbilical artery and found a close correlation. On the other hand, several authors reported some differences in the results of cultures from the two samples (Vidyasagar et al. 1970; Balagtas et al. 1971; Powers and Tooley 1972). In our series, cord blood culture was positive in one patient, while the peripheral blood was negative. It is now well accepted that peripheral blood cultures are necessary to evaluate the presence of systemic infection in patients under umbilical vessel catheterization.

The reported rates of septicemia associated with umbilical catheterization ranged from zero to several percent (Vidyasagar et al. 1970; Balagtas et al. 1971). The prevalence of septicemia in our study was $1 \%$. Thus, the septicemia associated with umbilical catheterization is never a negligible incidence. It must be emphasized that judicious surveillance for systemic infection is necessary for the patients under umbilical catheterization.

\section{References}

1) Anagnostakis, D., Kamba, A., Petrochilou, V., Arseni, A. \& Matsanitois, N. (1975) Risk of infection associated with umbilical vein catheterization. J. Pediat., 86, 759-765.

2) Balagtas, R.C., Bell, C.E., Edwards, L.D. \& Levin, S. (1971) Risk of local and systemic infections associated with umbilical vein catheterization: A prospective study in 86 newborn patients. Pediatrics, 48, 359-367.

3) Bard, H., Albert, G., Teasdale, F., Doray, B. \& Martineau, B. (1973) Prophylactic antibotics in chronic umbilical artery catheterization in respiratory distress syndrome. Arch. Dis. Childh., 48, 630-635.

4) Casalino, M.B. \& Lipsitz, P.J. (1971) Contamination of umbilical catheters. $J$. Pediat, , 78, 1077.

5) Cowett, R.M., Peter, G., Hakanson, D.O. \& Oh, W. (1976) Reliability of bacterial culture of blood obtained from an umbilical artery catheter. J. Pediat., 88, 1035-1036.

6) Daschner, F., Adam, D. \& Marget, W. (1974) Sepsis und Kontamination bei Nabelund Venenkathetern. Mschr. Kinderheilk, 122, 49-53.

7) Dunn, P.M. (1966) Localization of the umbilical catheter by post-mortem measurement. Arch. Dis. Childh., 41, 69-75.

8) Farichild, J.P., Graber, C.D., Vogel, E.H. \& Ingersoll, R.L. (1958) Flora of the umbilical stump. 2479 cultures. J. Pediat., 53, 538-546.

9) Franke, I., Loewenich, V. \& Koch, H. (1974) Infektiöse Komplikationen durch Katheterisierung der Nabelarterien. Mschr. Kinderheilk., 122, 488-489.

10) Johns, A.W., Kitchen, W.H. \& Leslie, D.W. (1972) Complications of umbilical vessel catheters. Med. J. Aust., 2, 810-815.

11) Kitterman, J.A., Phibbs, R.H. \& Tooley, W.H. (1970) Catheterization of umbilical vessels in newborn infants. Pediat. Clin. N. Amer., 17, 895-912.

12) Krauss, A.N., Albert, R.F. \& Kannan, M.M. (1970) Contamination of umbilical catheters in the newborn infant. J. Pediat., 77, 965-969.

13) Nordin, C.W. (1969) Application of antibiotics ointment to the site of venous catheterization - a controlled trial. J. infect. Dis., 120, 611-615.

14) Powers, W.F. \& Tooley, W.H. (1972) Contamination of umbilical vessel catheters: Encouraging information. Pediatrics, 49, 470-471. 
15) Symansky, M.R. \& Fox, H.A. (1972) Umbilical vessel catheterization: Indications, management, and evaluation of the technique. J. Pediat., 80, 820-826.

16) Vidyasagar, D., Downes, J.J. \& Boggs, T.R. (1970) Respiratory distress syndrome of newborn infants. II. Technic of catheterization of umbilical artery and clinical results of treatment of 124 patients. Clin. Pediat., 9, 332-337.

17) Zinner, S.H., Denny-Brown, B.C., Braun, P., Burke, J.P., Toala, P. \& Kass, E.H. (1969) Risk of infection with intravenous indwelling catheters: Effect of application of antibiotics ointment. J. infect. Dis., 120, 616-619. 